\title{
Nucleation and Initial Stages of Growth During the Atomic Layer Deposition (ALD) of Titanium Oxide on Mesoporous Silica
}

\author{
Wang Ke, ${ }^{1}$ Yang Liu, ${ }^{2}$ Xuelong Wang, ${ }^{3}$ Xiangdong Qin,,$\stackrel{1,}{ }$ Limei Chen, ${ }^{1}$ Robert M. Palomino, ${ }^{3}$ \\ Juan Pablo Simonovis, ${ }^{3,4}$ Ilkeun Lee, ${ }^{1}$ Iradwikanari Waluyo, ${ }^{4}$ José A. Rodriguez, ${ }^{3}$ Anatoly I. \\ Frenkel, ${ }^{2,3}$ Ping Liu, ${ }^{3}$ Francisco Zaera, ${ }^{1, *}$
}

1 Department of Chemistry, University of California, Riverside, CA 92521, USA

2 Department of Materials Science and Chemical Engineering, Stony Brook University, Stony Brook, NY 11794, USA

3 Chemistry Division, Brookhaven National Laboratory, Upton, NY 11973, USA

4 National Synchrotron Light Source II, Brookhaven National Laboratory, Upton, NY 11973, USA

† Present address: ASM America, Phoenix, AZ 85034, USA

* Corresponding author: zaera@ucr.edu

\section{Supplementary Material}




\section{Materials and Methods}

The titanium oxide films were deposited on commercial SBA-15 (ACS Material) using a homemade ALD reactor described in detail elsewhere, ${ }^{1}$ alternating exposures to TDMAT (tetrakis(dimethylamido)Ti(IV), Aldrich-Sigma, 99.999\% purity in a trace metals basis) and deionized water. Typical cycle times were TDMAT: $\mathrm{N}_{2}: \mathrm{H}_{2} \mathrm{O}: \mathrm{N}_{2}=20 \mathrm{~min}: 50 \mathrm{~min}: 2 \mathrm{~min}: 50 \mathrm{~min}$, and the depositions were carried out at a temperature of $375 \mathrm{~K}$, the same for both the sample and the reactor; the precursor was kept at $315 \mathrm{~K}$.

Adsorption-desorption isotherm measurements were carried out in a NOVA@2000e gas sorption system, using $\mathrm{N}_{2}$ as the adsorbent. Data analysis was carried out by using the Nova Win software: pore distributions and total pore volumes were estimated using the BJH (BarrettJoyner-Halenda) equations for Type IV isotherms, whereas total surface areas were calculated by using the BET (Brunauer-Emmett-Teller) isotherm.

Transmission Fourier transform infrared (FTIR) spectroscopy characterization experiments were performed on a Bruker Tensor 27 FTIR spectrometer equipped with a deuterated triglycine sulfate (DTGS) detector. About $10 \mathrm{mg}$ of the catalyst was pressed into a self-supporting wafer and loaded inside a homemade quartz cell with $\mathrm{NaCl}$ windows. ${ }^{2}$ The cell was evacuated and cooled down to $123 \mathrm{~K}$ (using liquid nitrogen), and IR spectra of the bare samples were acquired. Afterward, they were exposed to 20 Torr of CO (Matheson Tri-Gas, $\geq 99.9 \%$ purity) for $10 \mathrm{~min}$, and the cell was evacuated for $10 \mathrm{~min}$. IR spectra were recorded from 125 to $475 \mathrm{~K}$ at $10 \mathrm{~K}$ intervals as the sample and cell were warmed up (data not shown), and corrected using 
background spectra obtained under the same condition before adsorption.

Titanium loadings were quantified using a PerkinElmer Optima 7300DV ICP-AES apparatus. This system combines an SCD detector and an echelle optical system that enables the unit to measure all wavelengths simultaneously, in the ultraviolet wavelength range from 165 to $403 \mathrm{~nm}$ and in the visible wavelength range from 404 to $782 \mathrm{~nm}$.

Scanning transmission electron microscopy (STEM) images were obtained using a FEI Titan Themis 300 instrument equipped with a X-ray spectrometer for energy dispersion spectroscopy (EDX) imaging. The crystallinity of the samples was evaluated by X-ray diffraction (XRD), using a PANalytical Empyrean Series 2 diffractometer with $\mathrm{Cu} \mathrm{K} \alpha$ radiation $(\lambda=1.5406 \AA)$. The bandgap values were extracted from UV/visible diffuse reflectance spectra taken using a Varian Cary 500 double beam scanning UV/Vis/NIR spectrophotometer.

Most XPS data were collected with a commercial AP-XPS system (SPECS Surface Nano Analysis $\mathrm{GmbH}$, Germany) using a $\mathrm{Mg} \mathrm{K}_{a}$ anode (hv = $1253.6 \mathrm{eV}$ ) X-ray source, except for the survey traces, which were acquired using a Kratos analytical AXIS instrument equipped with a $165 \mathrm{~mm}$ mean radius semihemispherical electron energy analyzer and a monochromatized $\mathrm{Al} \mathrm{K} \mathrm{K}_{a}$ anode $(\mathrm{h} v=1486.7 \mathrm{eV}) \mathrm{X}$-ray source. ${ }^{3} \mathrm{Ti}_{2,3}$-edge NEXAFS data were collected at the IOS beamline (23-ID-2) of the NSLS-II facility of Brookhaven National Laboratory, following the total electron yield (TEY) by measuring the drain current. The photon flux was $10^{13}$ photons/s, and monocrystal gratings with 150,400 and 1200 lines $/ \mathrm{mm}$ were employed. The resolution was $\mathrm{E} / \Delta \mathrm{E}=104$, and the spot size (unfocused beam) $\sim 50 \mu \mathrm{m}$ (vertical) $\mathrm{x} \sim 150 \mu \mathrm{m}$ (horizontal). Ti 
K-edge X-ray absorption spectroscopy (XAS) data were acquired at the NSLS II 7-BM (QAS) beamline, in fluorescence mode using a PIPS detector. The data from a minimum of 30 scans (each scan was about 45 seconds long) were averaged to obtain each spectrum.

Solid-state ${ }^{29} \mathrm{Si} \mathrm{CP} / \mathrm{MAS}-\mathrm{NMR}$ spectra were acquired on a Bruker Avance 600 spectrometer, employing a cross-polarization contact time of $2 \mathrm{~ms}$, a ${ }^{1} \mathrm{H}$ decoupling bandwidth of $80 \mathrm{kHz}$, and a recycle time of $3 \mathrm{~s}$. Data were acquired as 12,000 co-added 2,048 complex data point FIDs with a $100 \mathrm{kHz}$ sweep width. Post acquisition processing consisted of exponential multiplication with $200 \mathrm{~Hz}$ of line broadening and zero filling to 4,096 data points. Chemical shifts were referenced to an external DSS sample.

DFT calculations were performed with the Vienna Ab Initio Simulation Package (VASP), ${ }^{4}$ using the projection-augmented wave (PAW) approach. ${ }^{5}$ The generalized gradient approximation (GGA) type exchange-correlation functional in the parametrization of Perdew, Burke, and Ernzerhof (PBE) was adopted. ${ }^{6}$ The energy cutoff for the basis set of wave functions was set to $500 \mathrm{eV}$. The k-mesh was generated at least denser than the density of one point per $0.05 \AA^{-3}$ with the Monkhorst-Pack method. ${ }^{7}$ For the relaxation of the model structures, the forces felt by each atom were well converged to a level smaller than $0.05 \mathrm{eV} / \AA$. For the DOS and bandgap calculations, the GGA+U method ${ }^{8}$ was used to address the strong correlation between the $3 \mathrm{~d}$ orbital electrons of Ti with an effective $\mathrm{U}$ value of $4.2 \mathrm{eV} .^{9,10}$ 


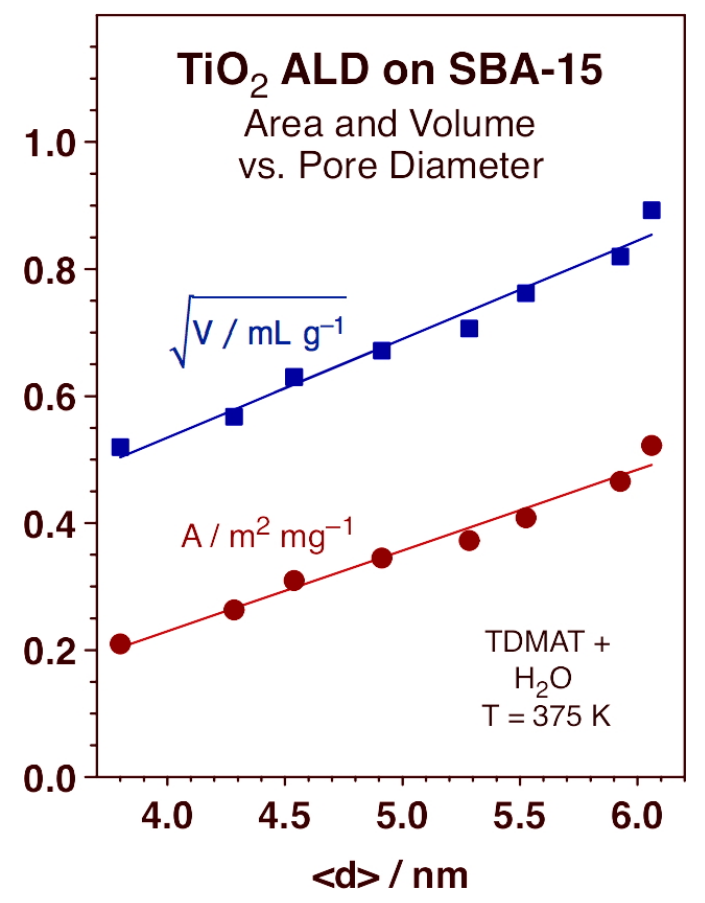

Figure S1. Total pore area (A; red solid circles) and square-root of the total pore volume $(\sqrt{\mathrm{V}}$; blue solid squares) of SBA-15 samples on which $\mathrm{TiO}_{2}$ films have been deposited as a function of average pore diameter $(<\mathrm{d}>)$, which decreases with increasing $\mathrm{TiO}_{2} \mathrm{ALD}$ cycles. These parameters are plotted as $\mathrm{A}$ and $\sqrt{\mathrm{V}}$ here to highlight the linear dependences that indicate film uniformity. 


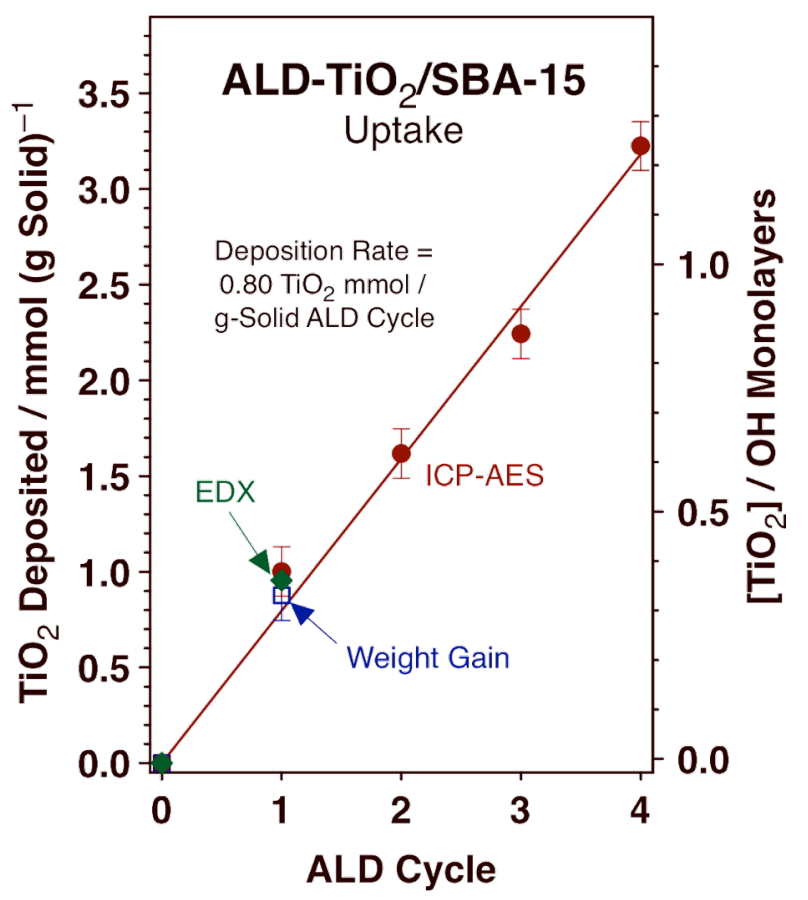

Figure S2. Mass gain (blue open square), EDX (green solid diamond), and ICP-AES (red solid circles) measurements of the uptake of $\mathrm{TiO}_{2}$ versus the number of ALD cycles used. The right-hand scale was derived from the direct monolayer calibration methods described in the text. 


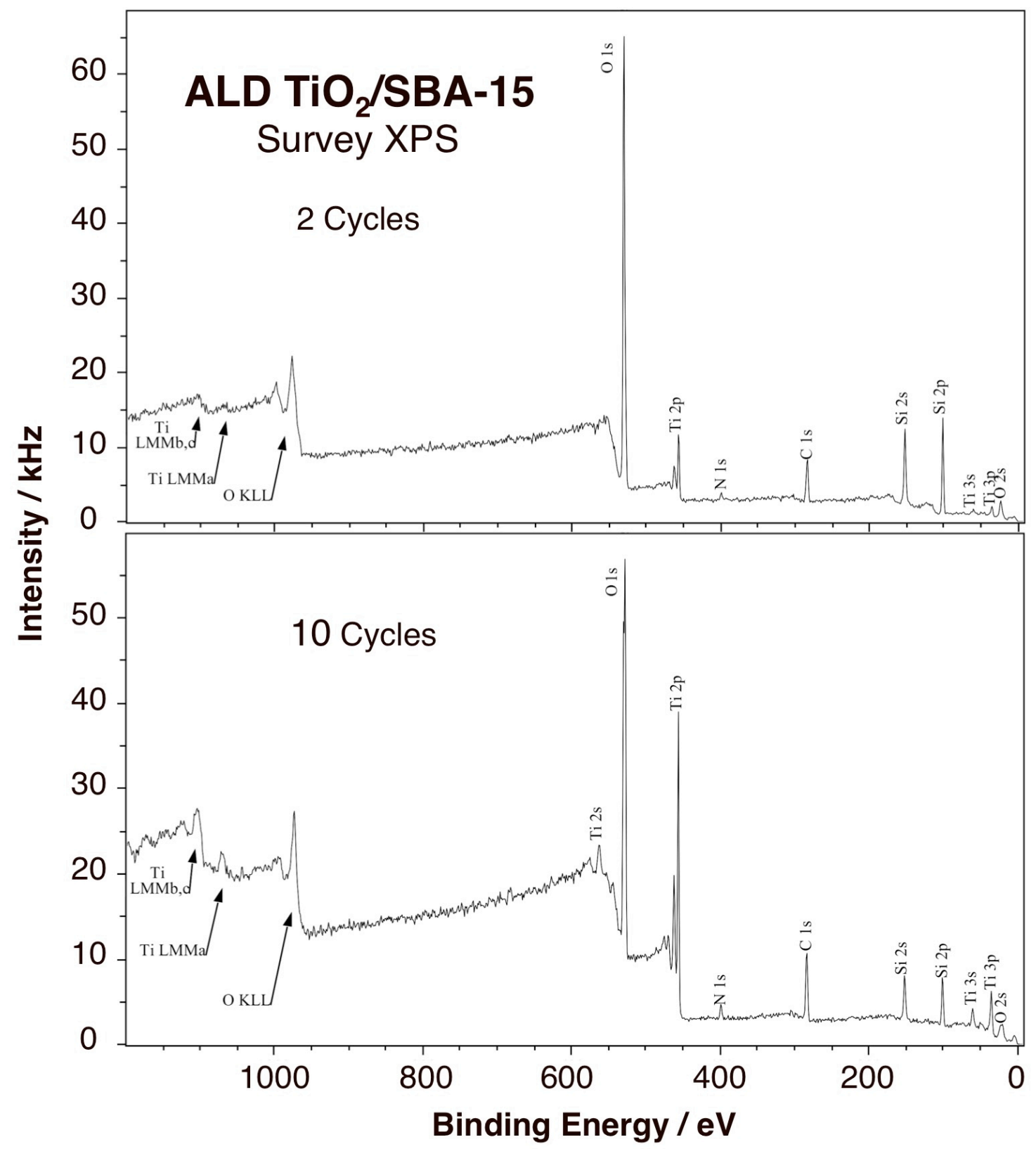

Figure S3. Survey XPS data for samples prepared using 2 (top) and 10 (bottom) $\mathrm{TiO}_{2}$ ALD on SBA15. Peaks are seen for the main elements expected, namely, $\mathrm{Si}, \mathrm{Ti}$, and $\mathrm{O}$, as well as for $\mathrm{N}$ and $\mathrm{C}$ impurities. The carbon is likely to originate from ex-situ handling of the solids, but a small amount of N, approximately 10 atom $\%$ with respect to the Ti coverage, seems to incorporate in the growing films during ALD. 

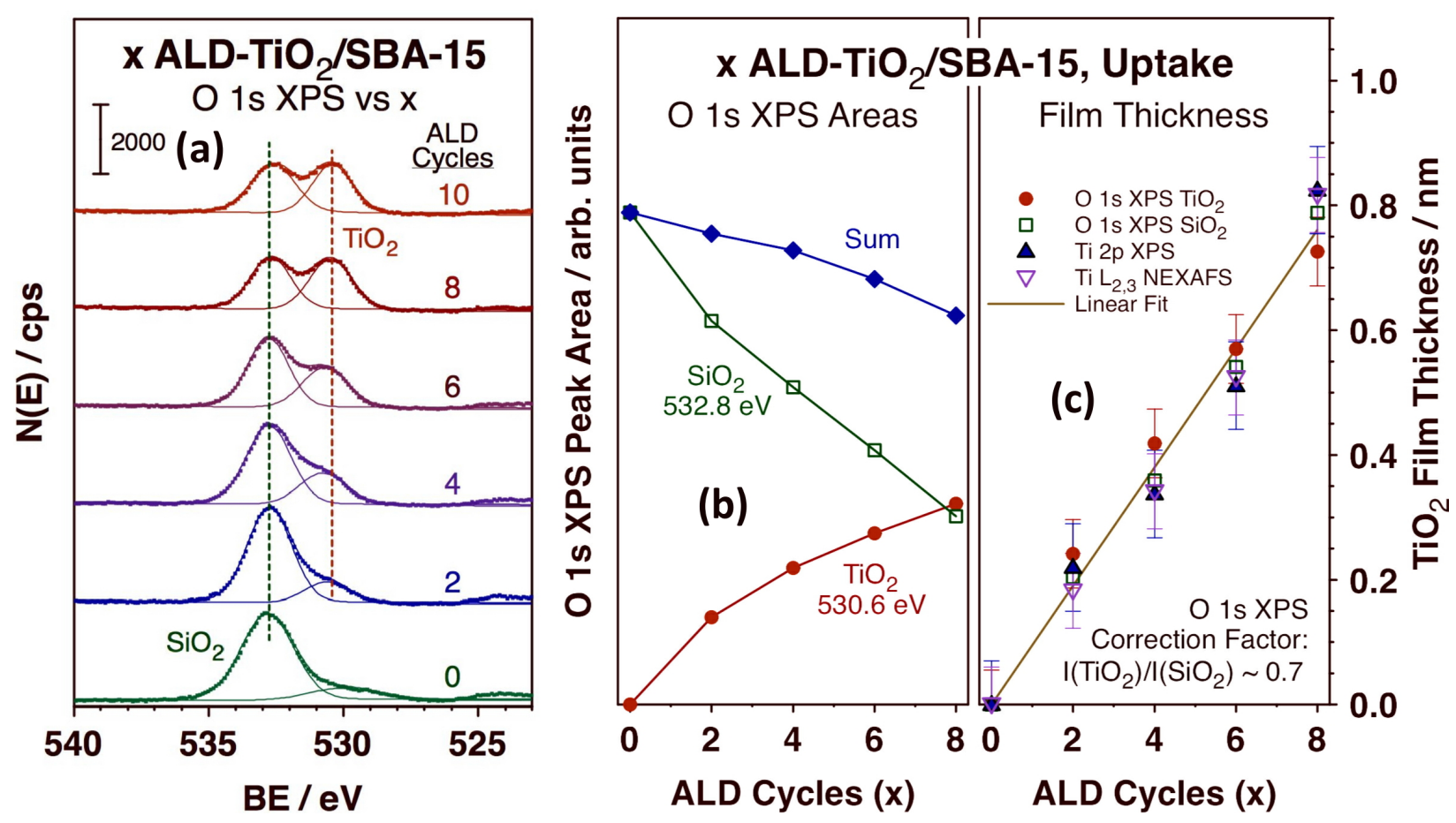

Figure S4. XPS data for samples prepared by $\mathrm{TiO}_{2}$ ALD on SBA-15. (a) O 1s XPS spectra after 0, 2, 4, 6, 8, and $10 \mathrm{TiO}_{2}$ ALD cycles. (b) $\mathrm{O}$ 1s XPS peak areas versus number of $\mathrm{TiO}_{2} \mathrm{ALD}$ cycles for the contributions from $\mathrm{SiO}_{2}(\mathrm{BE}=532.8 \mathrm{eV}$, green open squares $)$ and $\mathrm{TiO}_{2}(\mathrm{BE}=530.6 \mathrm{eV}$, red solid circles), as well as the total O 1 s XPS signal intensity (blue solid diamonds). (c) $\mathrm{TiO}_{2}$ film thickness versus number of $\mathrm{TiO}_{2}$ ALD cycles estimated from the areas of the O 1s XPS signals for $\mathrm{SiO}_{2}$ (red filled circles) and $\mathrm{TiO}_{2}$ (open green squares), the Ti 2p XPS peaks (spectra shown in Figure S5; blue solid uppointing triangles), and the $\mathrm{Ti} \mathrm{L}_{2,3}$ XANES data (spectra not shown; purple open downward-pointing triangles), assuming layer-by-layer growth and exponential evolution of the XPS and XAS signals with film thickness. The $\mathrm{O} 1 \mathrm{~s}$ XPS data corroborate the low density of the newly deposited $\mathrm{TiO}_{2}$ films: the number of oxygen atoms per unit volume within the $\mathrm{TiO}_{2}$ film is $\sim 2 / 3$ that of the underlying $\mathrm{SiO}_{2}$, as estimated by the slopes of the plots of the O 1s XPS signal intensities for $\mathrm{SiO}_{2}$ and $\mathrm{TiO}_{2}$, versus ALD cycles in Panel b. A deposition rate of approximately $0.95 \pm 0.20 \AA$ /cycle was estimated from these data. 


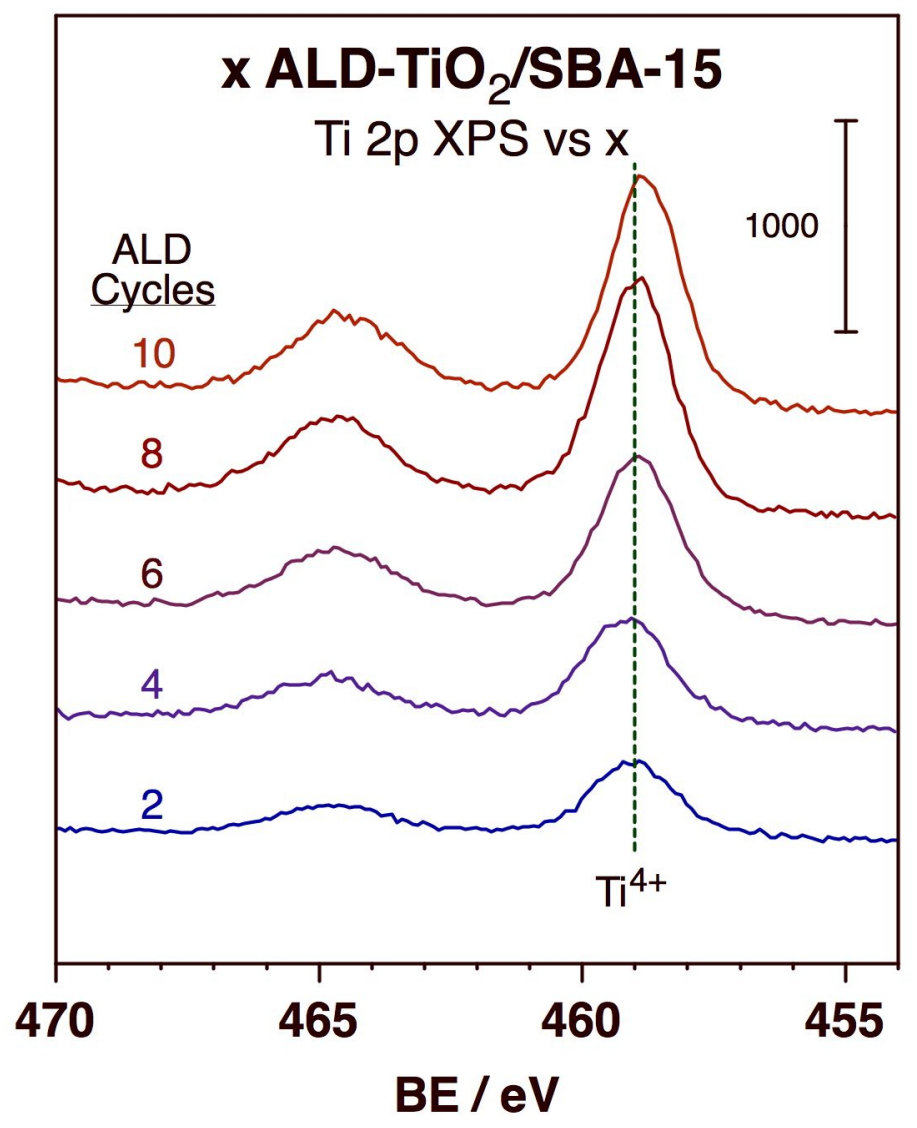

Figure S5. Ti $2 p$ XPS data for samples prepared by $\mathrm{TiO}_{2} \mathrm{ALD}$ on SBA-15 as a function of the number of ALD cycles used. Only peaks associated with fully oxidized $\mathrm{Ti}^{4+}$ are seen in all cases. 



Figure S6. (a) XRD data for the sample obtained after $10 \mathrm{TiO}_{2} \mathrm{ALD}$ cycles as a function of calcination temperature. Also shown are TEM images of the sample obtained after $10 \mathrm{TiO}_{2} \mathrm{ALD}$ cycles: (b) before calcination; (c) after calcination at $875 \mathrm{~K}$; (d) after calcination at $1075 \mathrm{~K}$; and (e) also after calcination at $1075 \mathrm{~K}$ (close up). The titanium oxide films are amorphous until calcination at or above $875 \mathrm{~K}$. Panel (e) shows that high-temperature calcination leads to crystallization via sintering and NP formation at the entrance of the pores 


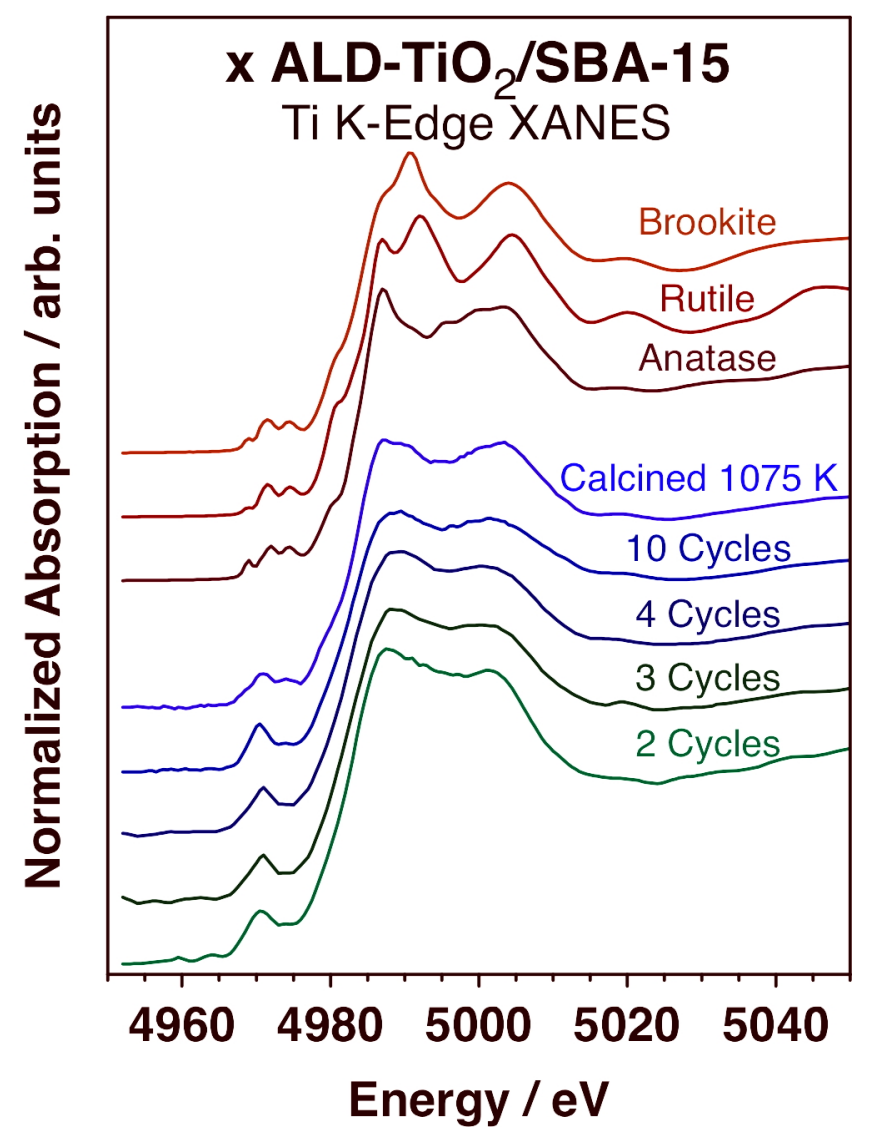

Figure S7. Ti K-edge XANES spectra for samples obtained after 2, 3, 4, and $10 \mathrm{TiO}_{2} \mathrm{ALD}$ cycles, as well as for the 10 -cycle sample after calcination to $1075 \mathrm{~K}$ and for anatase, rutile, and brookite crystalline reference samples. The data corroborate the amorphous nature of the deposited films unless they are calcined at high temperatures. Notice in particular the absence of the fine structure in the pre-edge region, for $\mathrm{E}<4976 \mathrm{eV}$, typical of the crystalline oxides. 


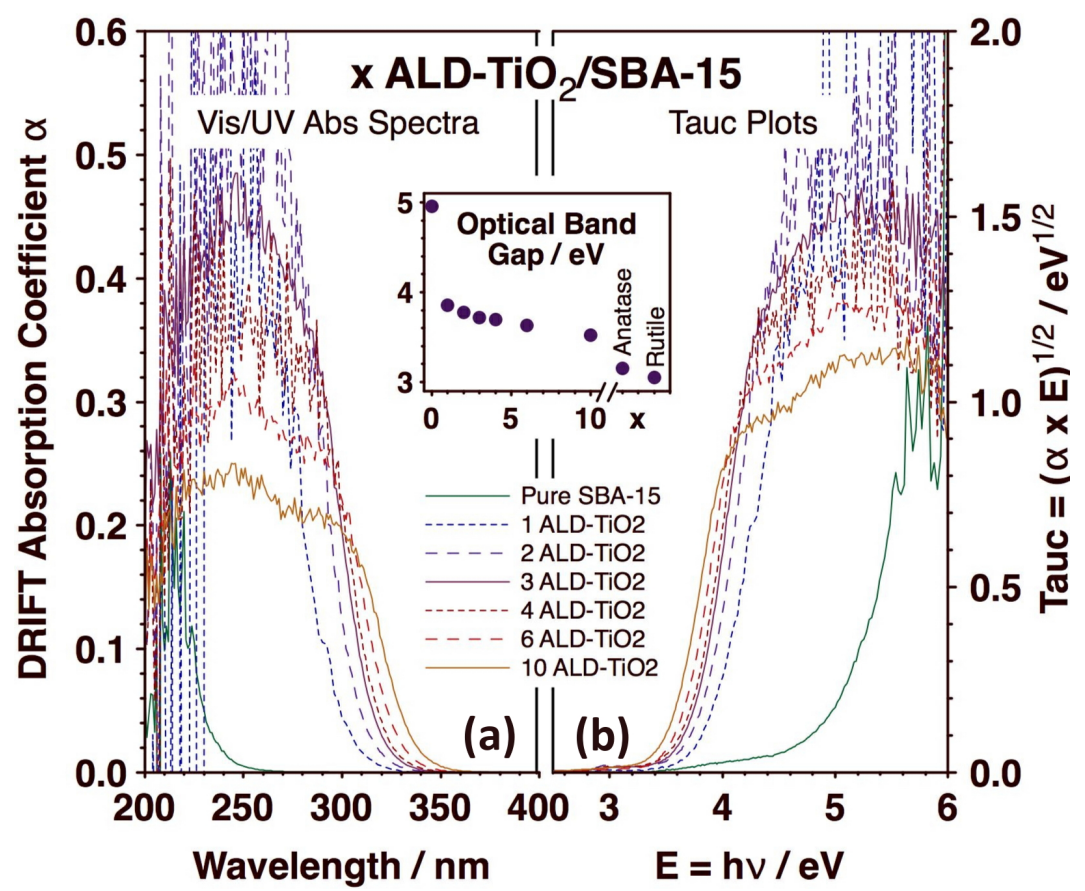

Figure S8. Diffuse-reflectance UV/visible absorption spectra from the samples obtained by $\mathrm{TiO}_{2}$ ALD on SBA-15 as a function of the number of cycles used. (a) Original data; and (b) Tauc plot. The bandgaps measured from these spectra, shown in the inset, are larger than those of crystalline $\mathrm{TiO}_{2}$, but they approach the bulk value as the film thickness increases. 

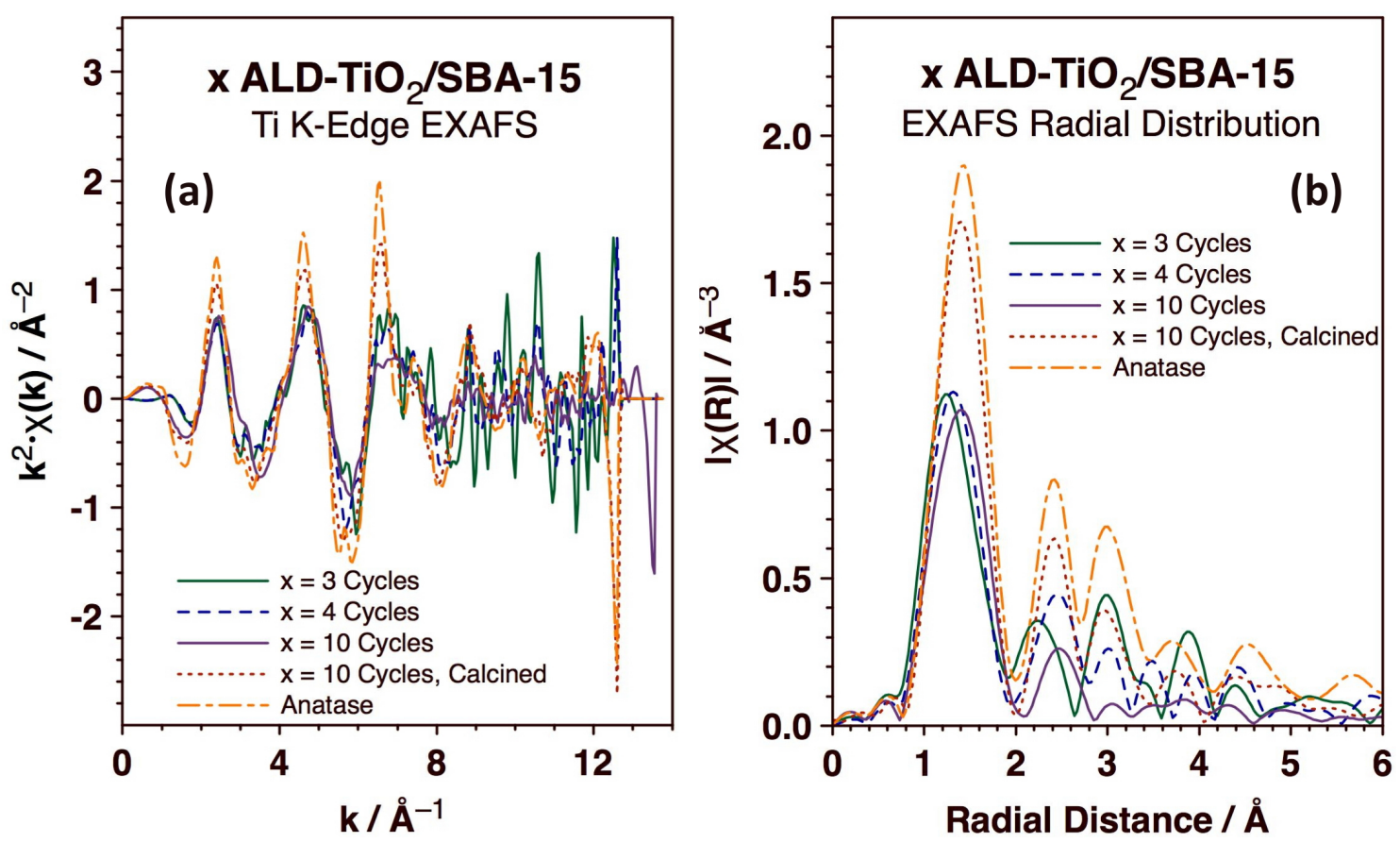

Figure S9. Ti K-edge EXAFS from the samples obtained by $\mathrm{TiO}_{2}$ ALD on SBA-15 as a function of the number of cycles used, for 3, 4, and 10 cycles, and after calcination of the 10 ALD-cycles sample. (a) Raw data; (b) Radial distribution. 
Table S1. Coordination numbers, average Ti-O bond distances and Debye Waller factors estimated from the EXAFS data for SBA-15 samples after $\mathrm{TiO}_{2}$ deposition using 3, 4, and 10 ALD cycles displayed in Figure S7. Data for the calcined film obtained after 10 ALD cycles and for anatase are provided for reference.

\begin{tabular}{l|c|c|c} 
Sample & $\mathbf{C N}$ & $<\mathbf{d}(\mathbf{T i}-\mathbf{O}) / \AA>$ & $\boldsymbol{\sigma}^{2} \mathbf{( T i - O )} / \AA^{2}$ \\
\hline $3 \mathrm{TiO}_{2}$ ALD cycles/SBA-15 & $3.4 \pm 1.7$ & $1.856 \pm 0.019$ & $0.0051 \pm 0.0066$ \\
$4 \mathrm{TiO}_{2}$ ALD cycles/SBA-15 & $3.0 \pm 1.6$ & $1.873 \pm 0.043$ & $0.0032 \pm 0.0064$ \\
$10 \mathrm{TiO}_{2}$ ALD cycles/SBA-15 & $3.2 \pm 0.8$ & $1.938 \pm 0.023$ & $0.0073 \pm 0.0036$ \\
& & & \\
$10 \mathrm{TiO}_{2}$ ALD cycles/SBA-15 Calcined & 6 (Fixed) & $1.941 \pm 0.015$ & $0.0077 \pm 0.0012$ \\
Anatase & 6 (Fixed) & $1.956 \pm 0.017$ & $0.0067 \pm 0.0028$
\end{tabular}


Table S2. Coordination numbers, average Ti-O bond distances and Debye Waller factors estimated from EXAFS data for SBA-15 samples after $\mathrm{TiO}_{2}$ deposition using 4 and $10 \mathrm{ALD}$ cycles. In this set, fitting of the data for $\mathrm{Ti}-\mathrm{O}$ first coordination was complemented with a second coordination sphere where only Si next-near neighbors were considered.

\begin{tabular}{|c|c|c|c|c|c|c|}
\hline \multirow[b]{2}{*}{ Sample } & \multicolumn{3}{|c|}{ 1st Coordination Sphere (Ti-O) } & \multicolumn{3}{|c|}{ 2nd Coordination Sphere (Ti-Si) } \\
\hline & $\mathbf{C N}$ & $<\mathbf{d}>/ \AA$ & $\sigma^{2} / \AA^{2}$ & $\mathbf{C N}$ & $<\mathbf{d}>/ \AA$ & $\sigma^{2} / \AA^{2}$ \\
\hline 4 ALD cycles & $3.2 \pm 1.4$ & $\begin{array}{c}1.901 \pm \\
0.014\end{array}$ & $\begin{array}{c}0.0048 \pm \\
0.0025\end{array}$ & $1.8 \pm 1.4$ & $\begin{array}{c}2.87 \pm \\
0.06\end{array}$ & $\begin{array}{c}0.004 \pm \\
0.010\end{array}$ \\
\hline 10 ALD cycles & $3.3 \pm 0.6$ & $\begin{array}{c}1.953 \pm \\
0.016\end{array}$ & $\begin{array}{c}0.0080 \pm \\
0.0025\end{array}$ & $1.6 \pm 0.9$ & $\begin{array}{c}3.04 \pm \\
0.06\end{array}$ & $\begin{array}{c}0.011 \pm \\
0.008\end{array}$ \\
\hline
\end{tabular}

Table S3. Coordination numbers, average Ti-O bond distances and Debye Waller factors estimated from EXAFS data for SBA-15 samples after $\mathrm{TiO}_{2}$ deposition using 4 and 10 ALD cycles. In this set, fitting of the data for $\mathrm{Ti}-\mathrm{O}$ first coordination was complemented with a second coordination sphere where only Ti next-near neighbors were considered.

\begin{tabular}{l|c|c|c|c|c|c} 
& \multicolumn{3}{|c|}{ 1st Coordination Sphere (Ti-O) } & \multicolumn{4}{|c}{ 2nd Coordination Sphere (Ti-Ti) } \\
Sample & $\mathbf{C N}$ & $<\mathbf{d}>/ \AA$ & $\mathbf{\sigma}^{\mathbf{2}} / \AA^{\mathbf{2}}$ & $\mathbf{C N}$ & $<\mathbf{d}>/ \AA$ & $\mathbf{\sigma}^{\mathbf{2}} / \AA^{\mathbf{2}}$ \\
\hline 4 ALD cycles & $3.3 \pm 1.2$ & $1.903 \pm$ & $0.0050 \pm$ & $2.7 \pm 2.4$ & $3.00 \pm$ & $0.010 \pm$ \\
& & 0.012 & 0.0050 & & 0.07 & 0.010 \\
\multirow{2}{*}{10 ALD cycles } & $3.4 \pm 0.6$ & $1.952 \pm$ & $0.0083 \pm$ & $2.8 \pm 2.4$ & $3.04 \pm$ & $0.018 \pm$ \\
& & 0.015 & 0.0025 & & 0.06 & 0.009
\end{tabular}


(a) $\beta$-Cristobalite $\mathrm{SiO}_{2}(001)$
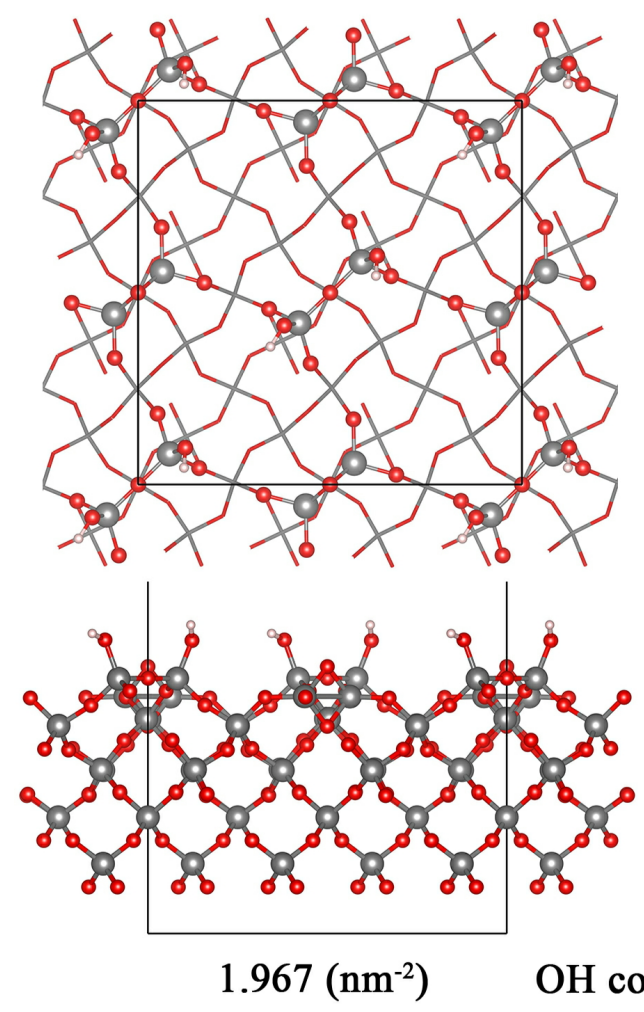

(b) $\beta$-Cristobalite $\mathrm{SiO}_{2}(110)$

(c)
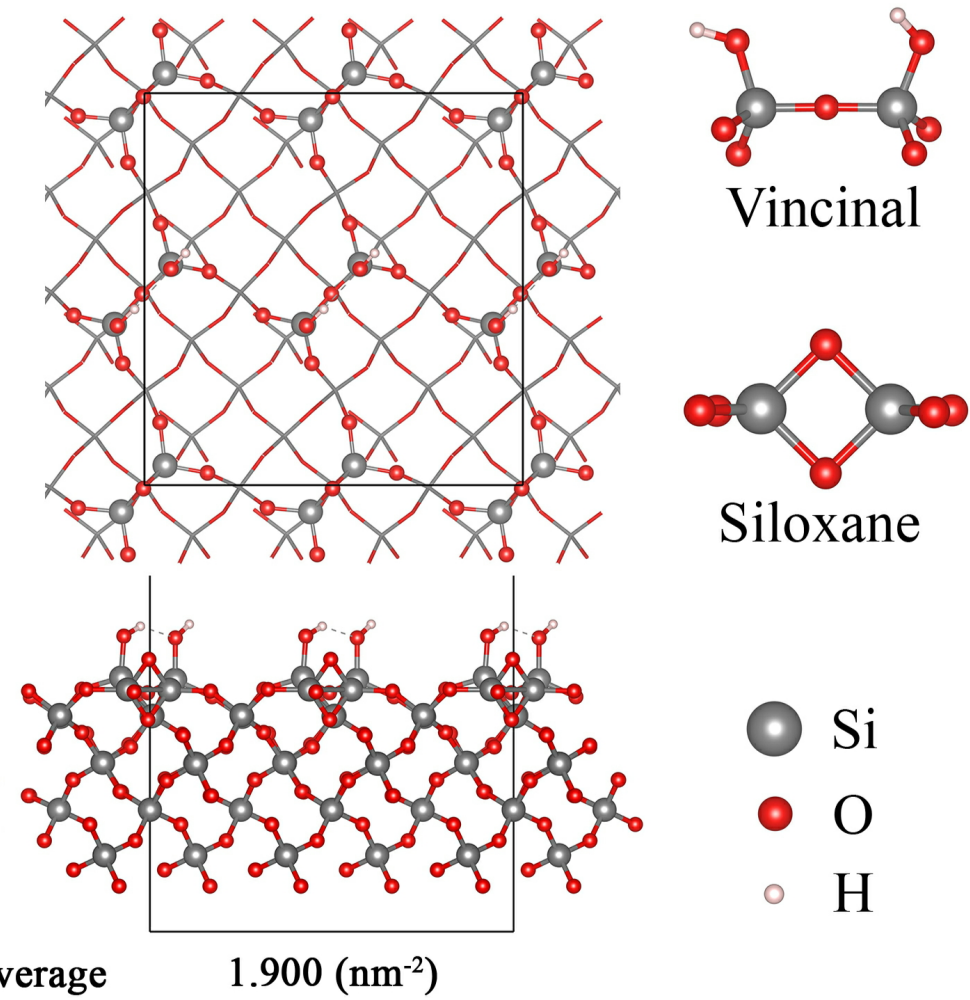

Figure S10. Structural model used in our DFT calculation for the underlying $\mathrm{SiO}_{2}$ substrate. Two different planes of $\beta$-crystobalite were used, (001) (a); and (110) (b). In order to reach a surface silanol coverage close to that measured experimentally for SBA-15, some positions were terminated with either vincinal or siloxane moieties (c). 

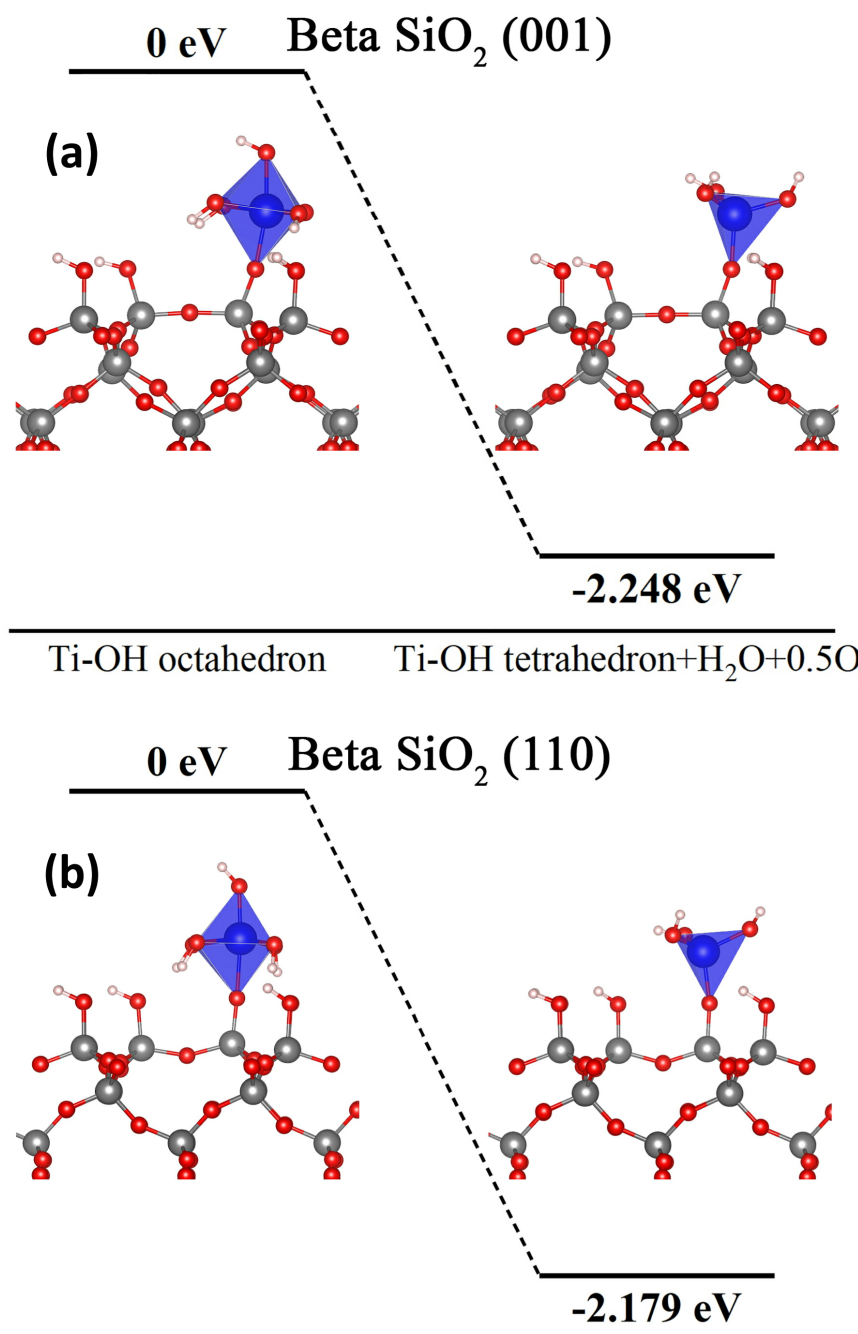

Ti-OH octahedron Ti-OH tetrahedron $+\mathrm{H}_{2} \mathrm{O}+0.5 \mathrm{O}_{2}$

Figure S11. Energetics of the formation of tetrahedral versus octahedral individual $\operatorname{TiO}_{x}(\mathrm{OH}) \mathrm{y}$ units on the Si-OH nucleation centers. Tetrahedral structures are clearly preferred. 

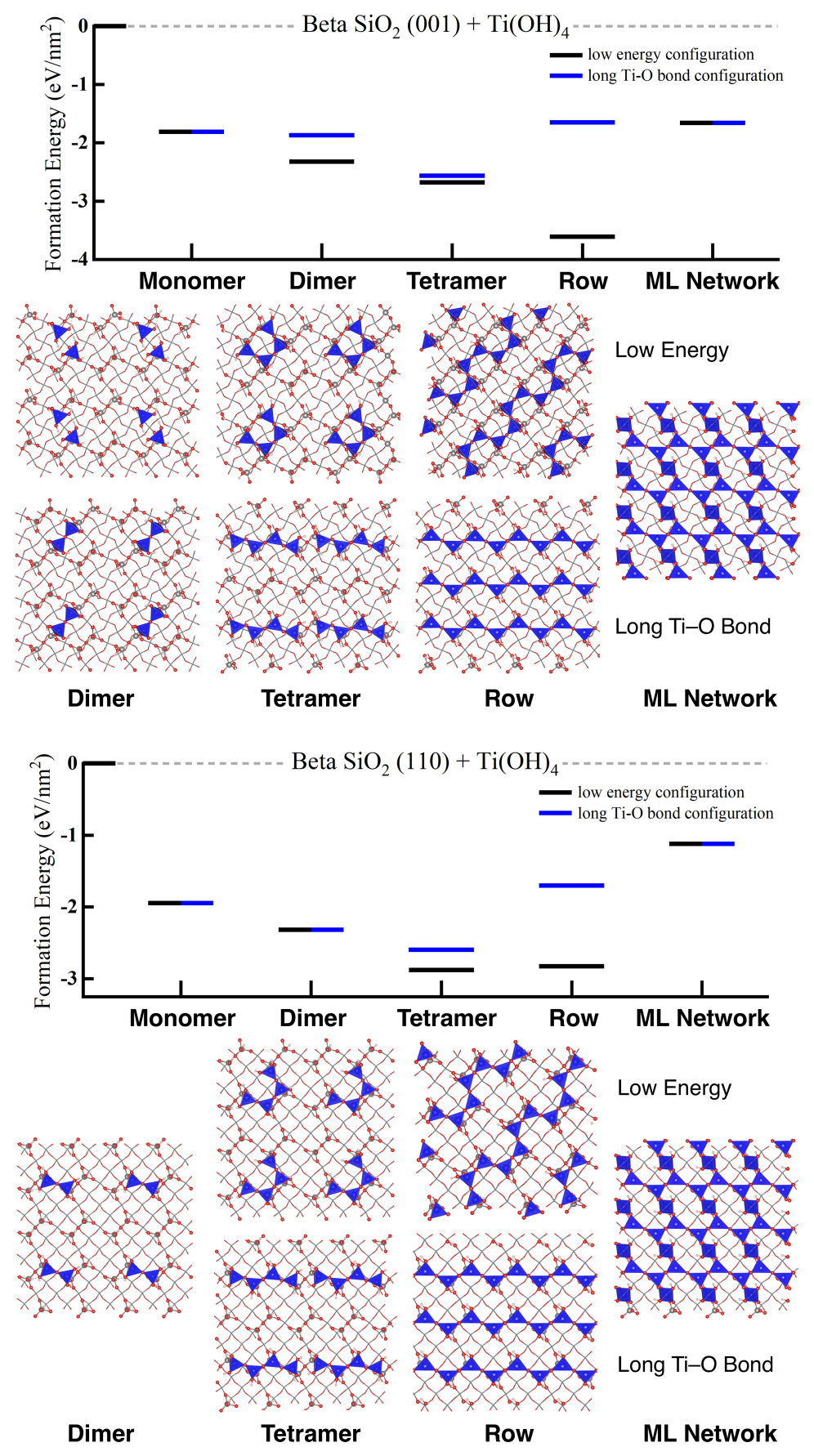

Figure S12. Energetics of the formation of the $\mathrm{TiO}_{\mathrm{x}}(\mathrm{OH}) \mathrm{y}$ layers on the $\mathrm{Si}-\mathrm{OH}$ surface. Structures and energetics are provided for the build up of the titanium oxide first layer via the sequential deposition of monomers, dimers, tetramers, rows, and monolayer (ML) networks. Two sets of data are provided, for the (001) (top) and (110) (bottom) $\beta$-crystobalite surfaces, and within each sets two rows of results are reported, for the lowest energy (top, black bars) and longest $\mathrm{Ti}-\mathrm{O}$ bond length (bottom, blue bars) structures of the titanium oxide overlayer. 


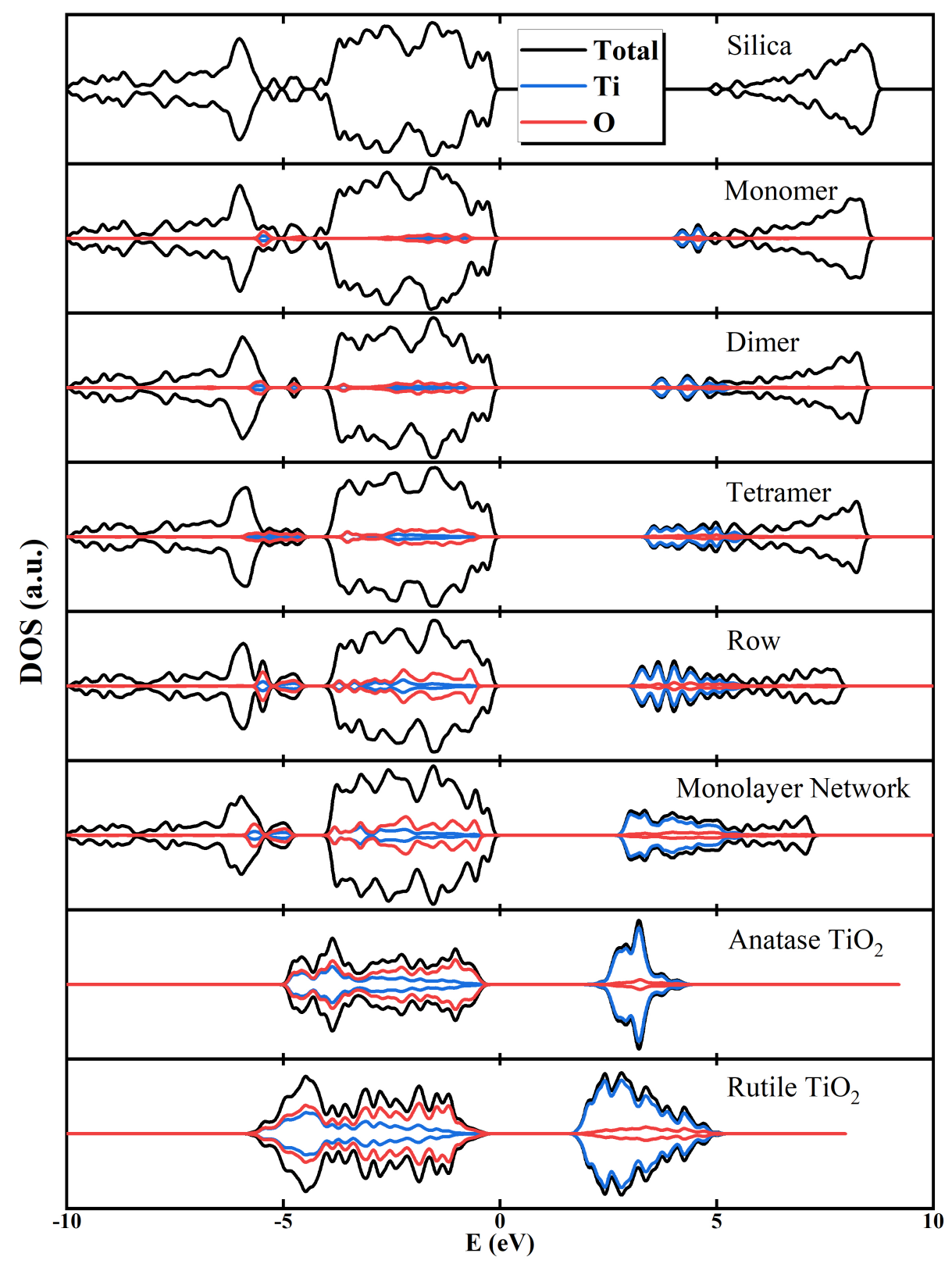

Figure S13. Total density of states (DOS) and elemental-decomposed DOS, estimated by DFT, of the bare $\mathrm{SiO}_{2}(001)$ surface (top panel), $\mathrm{SiO}_{2}(001)$ surfaces after deposition of $\mathrm{TiO}_{\mathrm{x}}(\mathrm{OH})_{\mathrm{y}}$ monomers, dimers, tetramers, rows, and network layers (2nd to 6th panels), and bulk $\mathrm{TiO}_{2}$ anatase and rutile crystals (7th and 8th panels). 


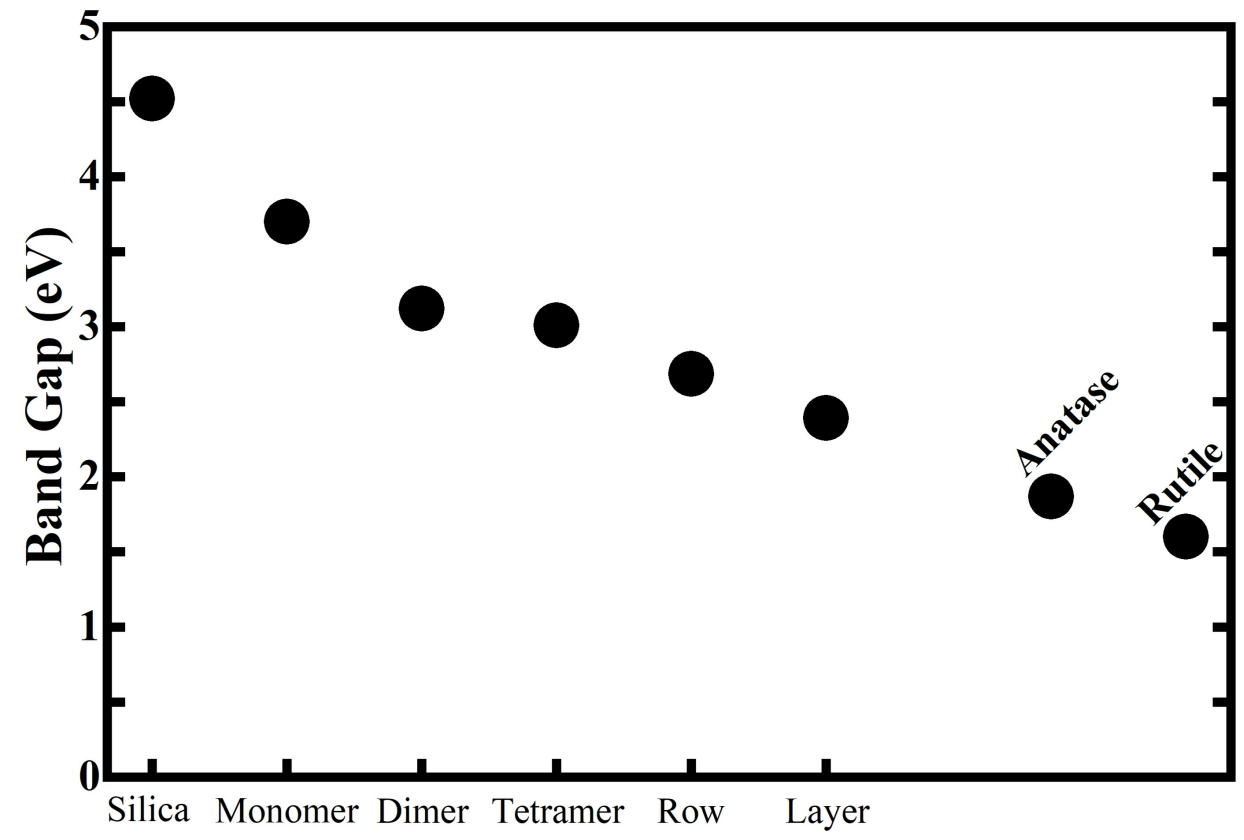

Figure S14. Bandgaps calculated from the DOS data in Figure S13. The DFT+U calculations underestimate the bandgap of $\mathrm{TiO}_{2}$ materials, a well known problem already reported in the literature, ${ }^{11}$ but nicely reproduce qualitatively the trend in Figure S8, with the bandgap decreasing as the size of the $\mathrm{TiO}_{\mathrm{x}}(\mathrm{OH}) \mathrm{y}$ clusters increases and approaching that of bulk $\mathrm{TiO}_{2}$ materials. 


\section{References}

(1) Weng, Z.; Chen, Z.-h.; Qin, X.; Zaera, F. Sub-Monolayer Control of the Growth of Oxide Films on Mesoporous Materials. J. Mater. Chem. A 2018, 6, 17548-17558.

(2) Cao, Y.; Chen, B.; Guerrero-Sánchez, J.; Lee, I.; Zhou, X.; Takeuchi, N.; Zaera, F. Controlling Selectivity in Unsaturated Aldehyde Hydrogenation Using Single-Site Alloy Catalysts. ACS Catal. 2019, 9, 9150-9157.

(3) Joo, J. B.; Dillon, R.; Lee, I.; Yin, Y.; Bardeen, C. J.; Zaera, F. Promotion of Atomic Hydrogen Recombination as an Alternative to Electron Trapping for the Role of Metals in the Photocatalytic Production of $\mathrm{H}_{2}$. Proc. Natl. Acad. Sci. U. S. A. 2014, 111, 79427947.

(4) Kresse, G.; Furthmüller, J. Efficiency of Ab-Initio Total Energy Calculations for Metals and Semiconductors Using a Plane-Wave Basis Set. Computational Materials Science 1996, $6,15-50$.

(5) Blöchl, P. E. Projector Augmented-Wave Method. Phys. Rev. B 1994, 50, 17953-17979.

(6) Perdew, J. P.; Burke, K.; Ernzerhof, M. Generalized Gradient Approximation Made Simple. Phys. Rev. Lett. 1996, 77, 3865-3868.

(7) Monkhorst, H. J.; Pack, J. D. Special Points for Brillouin-Zone Integrations. Phys. Rev. B 1976, 13, 5188-5192.

(8) Anisimov, V. I.; Aryasetiawan, F.; Lichtenstein, A. I. First-Principles Calculations of the Electronic Structure and Spectra of Strongly Correlated Systems: The Lda+U Method. J. Phys.: Condens. Matter 1997, 9, 767-808. 
(9) Morgan, B. J.; Watson, G. W. A Density Functional Theory + U Study of Oxygen Vacancy Formation at the (110), (100), (101), and (001) Surfaces of Rutile $\mathrm{TiO}_{2}$. J. Phys. Chem. C 2009, 113, 7322-7328.

(10) Pham, H. H.; Wang, L.-W. Oxygen Vacancy and Hole Conduction in Amorphous $\mathrm{TiO}_{2}$. Phys. Chem. Chem. Phys. 2015, 17, 541-550.

(11) Ghuman, K. K.; Singh, C. V. Effect of Doping on Electronic Structure and Photocatalytic Behavior of Amorphous $\mathrm{TiO}_{2}$. J. Phys.: Condens. Matter 2013, 25, 475501. 